\title{
Development of a cost-effective wastewater treatment process: combination of different process
}

\author{
Song $\mathrm{Du}^{1, \mathrm{a}}$, Wenbiao $\mathrm{Jin}^{1}$ \\ ${ }^{1}$ Harbin Institute of Technology Shenzhen Graduate School, Shenzhen Engineering Laboratory of Microalgal Bioenergy, Shenzhen, \\ 518055, P. R. China.
}

\begin{abstract}
Caprolactam wastewater produced by the production process of caprolactam is characterized by a very high toxicity and chemical oxygen demand (COD) values, having potential harm to the environment if treated improperly. However, these characteristics make caprolactam wastewaters difficult to treat using traditional methods. So the aim of this work was to develop a cost-effective caprolactam wastewater treatment process. Fenton oxidation, sequencing batch reactor activated sludge process (SBR) and electro-catalytic oxidation were proposed to treat caprolactam wastewater in the laboratory scale, and the treatment effects were investigated. Compared with Fenton oxidation, SBR and electro-catalytic oxidation can treat caprolactam wastewater at a lower cost and more efficiently. The pilot test results indicate that the COD can be decreased to less than $1000 \mathrm{mg} / \mathrm{L}$ by the combination process, and when the COD removal rates maintain $90 \%$, the cost of caprolactam wastewater treatment is below $6 \mathrm{yuan} / \mathrm{m}^{3}$. The combination process showed better economic benefit.
\end{abstract}

\section{Introduction}

Caprolactam is a monomer used for synthesizing nylon fiber (nylon-6), which is generally used in polymer materials $[1,2]$. At present, cyclohexanone-hydroxylamine (hydroxylamine-phosphate-oxime) is the most widely method used in the industrial production of caprolactam in the world. However, a lot of organic wastewater will be produced in the production process. What's worse, the wastewater contains various high concentration organic compounds, such as cyclohexanone, cyclohexane, benzene, toluene, cyclohexanone oxime, organic acid and caprolactam, which impart high chemical oxygen demand (COD) and toxicity[3]. Hence, the treatment of this wastewater is difficult.

Fenton oxidation method is suitable for the treatment of high concentration organic wastewater, which generating free radicals $(\cdot \mathrm{OH})$ with strong oxidizing power by the action of $\mathrm{H}_{2} \mathrm{O}_{2}$ and $\mathrm{Fe}^{2+}$, initiating and propagating free radical chain reactions to accelerate the oxidation of organic and reducing substances. Wang used this method to treat caprolactam wastewater, the COD removal rates reached $70 \%$, and biodegradability of wastewater was improved. But its high cost makes it difficult to be widely used. In recent years, SBR has been successfully employed to remove COD and organic matters from wastewaters[4]. Generally, the $\mathrm{BOD}_{5} / \mathrm{COD}$ index $(\mathrm{B} / \mathrm{C})$ was used to assess the wastewater biodegradability, and when the value of $\mathrm{B} / \mathrm{C}$ exceeded 0.3 in wastewater, meaning that wastewater can be biochemically treated. Studies have confirmed that some organic matter of caprolactam wastewater is biodegradable, such as caprolactam toluene, benzoic acid and acetic acid, of which the $\mathrm{B} / \mathrm{C}$ were all above 0.5 [5]. However, it is difficult to meet the wastewater discharge standards only using the SBR process. As we know, with the continuous deepening of electrochemical theory, electro-catalytic process has been widely used in the treatment of various kinds of wastewater, which can be carried out at normal temperature and pressure, and the reactor equipment is small in scale and easy to operate. Using $\mathrm{Ti} / \mathrm{SnO}_{2}-\mathrm{Sb}_{2} \mathrm{O}_{3}-\mathrm{CeO}_{2}$ as the electrode to treat caprolactam wastewater, the removal rate of COD reached $50 \%$. However, it also has the problem with high processing cost. Therefore, whether a combination of different processes can treat caprolactam wastewater costeffectively, is worth our study. In order to develop a costeffective caprolactam wastewater treatment combi- nation process, this study not only investigates the treatment effect of Fenton oxidation, SBR and electro- catalytic process, but also explores the treatment effect of combination process and evaluates the cost of wastewater treatment, which providing new ideas and methods for the treatment of caprolactam wastewater.

\section{Materials and methods}

\subsection{Wastewater}

The wastewater was obtained from a state-owned chemical factory (Zhejiang, China). The physicochemical characteristics of the wastewater are given in Table 1. The wastewater is yellow-brown due to various organic and

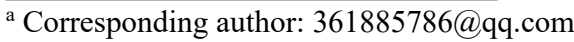


inorganic compounds, has high conductivity 6930- 7940 $\mu \mathrm{S} / \mathrm{cm}$ and COD $2854-6359 \mathrm{mg} / \mathrm{L}$. The effluent is highly alkaline, $\mathrm{pH}$ 11.6-12.1.

Table 1. Physicochemical Characteristics of the wastewater from caprolactam production workshop

\begin{tabular}{cc}
\hline Parameter & Value \\
\hline $\mathrm{pH}$ & $11.6-12.1$ \\
Conductivity $(\mu \mathrm{S} / \mathrm{cm})$ & $6930-7940$ \\
Chemical oxygen demand $(\mathrm{COD}, \mathrm{mg} / \mathrm{L})$ & $2854-6359$ \\
Sulfate ion $\left(\mathrm{SO}_{4}{ }^{2-}, \mathrm{mg} / \mathrm{L}\right)$ & $131-165$ \\
Ammonia nitrogen $\left(\mathrm{NO}_{3}{ }^{-\mathrm{N}}, \mathrm{mg} / \mathrm{L}\right)$ & $48.4-53.5$ \\
Total nitrogen $(\mathrm{TN}, \mathrm{mg} / \mathrm{L})$ & $1540-1954$ \\
\hline
\end{tabular}

\subsection{Analysis Methods}

The concentrations of COD were determined according to the dichromate method [6]. The $\mathrm{pH}$ of the solution was measured by a $\mathrm{pH}$ meter (Beijing Chemical Pharmaceutical Instrument Co. Ltd, China). The concentrations of SO42- were determined by ion chromatography. NH3-N concentrations of the samples was determined by using standard methods, and the equipment was Agilent Cary 100. Total nitrogen (TN) was measured by TOC analyzer (Elementar, German).

The COD removal rate was calculated through Eq. (1):

$$
\text { COD removal rate }=\frac{\left(C_{0}-C_{t}\right)}{C_{0}} \times 100 \%
$$

where $\mathrm{C} 0$ is the initial COD value of raw wastewater and $\mathrm{Ct}$ is the concentration of COD of the effluents at time $\mathrm{t}$.

\subsection{Fenton Oxidation Laboratory-scaled Experi- ment}

The Fenton oxidation experiment was conducted with a 5 $\mathrm{L}$ reactor independently developed by the wastewater treatment laboratory of China Coal Research Institute (CCRI), the diagram of the reactor is shown in Figure 1.

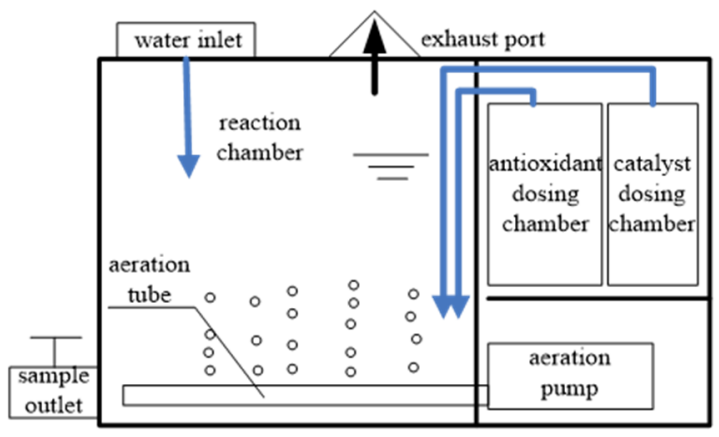

Figure 1. Diagram of Fenton oxidation reactor

The experiment went like this: First, the $\mathrm{pH}$ of wastewater needs to be adjusted between 3.5 and 4.0 by concentrated sulfuric acid $(10 \mathrm{~mol} / \mathrm{L})$ before the water was transferred to the reaction chamber. Then, H2O2 (mass fraction is $30 \%$ ) and $\mathrm{FeSO} 4$ (mass fraction is 3\%o) respectively as the oxidant and catalyst were added into wastewater of reaction chamber, and the aeration was carried out in the bottom of reaction chamber by the aeration tube. Subsequently, the wastewater samples were collected every 30 minutes during the reaction, and the $\mathrm{pH}$ of wastewater samples were adjusted to 10 by sodium hydroxide solution $(1 \mathrm{~mol} / \mathrm{L})$, taking place precipitation reaction. After the precipitation, supernatant was extracted and the $\mathrm{pH}$ was adjusted to neutral. Finally, the concentration of COD was analyzed $[7,8]$. The oxidant dosage of each batch experiment is $2 \%, 5 \%, 1 \%, 2 \%$, and $3 \%$ of the total volume of water, respectively.

\subsection{SBR Laboratory-scaled Experiment}

The SBR laboratory-scaled experiment reactor, cylindrical $(\varnothing 10 \mathrm{~cm} \times 30 \mathrm{~cm})$, the effective volume about $2.0 \mathrm{~L}$ (Figure 2), was made of transparent synthetic glass, and there was a stirrer inside, which was used to enhance the efficiency of denitrification by stirring and mixing wastewater when aeration was stopped. There are multiple sampling ports at different heights on the side of the main body. Aeration holes are provided at the bottom for aeration, and a vent valve is designed for air defense and mud discharge.

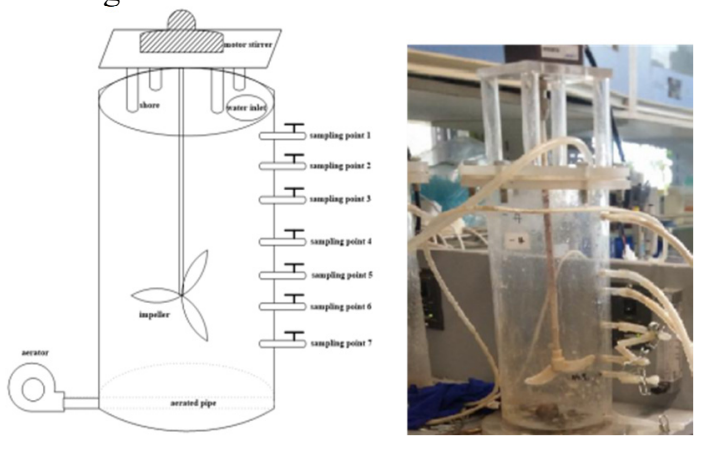

Figure 2. Diagram of SBR device

The reactor inoculation sludge was taken from the activated sludge in Tsinghua University campus sewage treatment station. SBR experiment was performed as follows: At first, a certain amount of activated sludge was taken and diluted to $2 \mathrm{~L}$ with tap water to ensure that settling velocity (SV) was about $30 \%$. After still and subsiding for $30 \mathrm{~min}$, remove $500 \mathrm{ml}$ supernatant and add $500 \mathrm{~mL}$ neutral $\mathrm{pH}$ mixed wastewater for keeping wastewater at $2 \mathrm{~L}$. Whereafter, continuing aeration for 20 $\mathrm{h}$, then still and subsiding for $30 \mathrm{~min}$ again, remove 500 $\mathrm{mL}$ supernatant and add neutral $\mathrm{pH}$ mixed wastewater to keep wastewater at $2 \mathrm{~L}$, continue to aerate, and circulate the process for $15 \mathrm{~d}$ to culture microorganisms. After the effluent was stable, take the supernatant every other day to measure COD concentration.

\subsection{Electro-catalytic Laboratory-scaled Experi- ment}

The electro-catalytic experiment used the three-dimensional electrode reactor invented by Tsinghua University. 
Granular and clastic electrode materials were filled between battery lead plates of the three-dimensional electrode reactor. Because of forming new electrodes when these electrode materials electrified charged under the induction of anodic and cathodic electrode power plants, the electrochemical reaction area was extended from surface of the counter electrode to surface of these electrode materials, the active specific surface of electrochemical reaction was considerably increased and the mass transfer distance was shortened, enhancing the efficiency of degrading contaminant. Principle of electrocatalytic reaction is shown in Figure 3. At the anode, $\mathrm{H} 2 \mathrm{O}$ was broken up into hydroxyl radical $(\mathrm{OH}-)$ and $\mathrm{H}+$, the hydroxyl radical $(\mathrm{OH}-)$ reacted with organics in the wastewater to generate carbon dioxide, and $\mathrm{H}+$ generated $\mathrm{H} 2$ at the cathode[9].

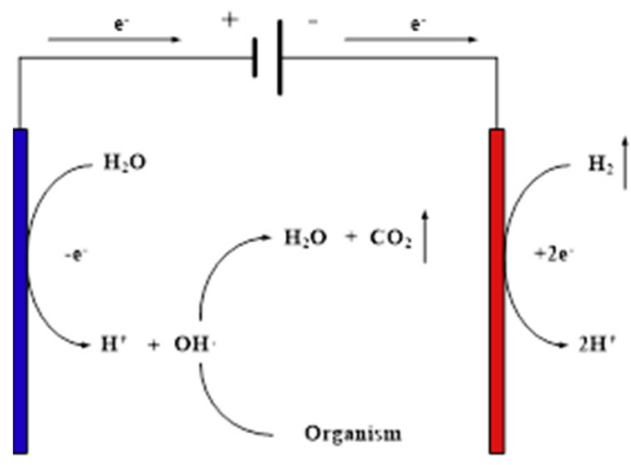

Figure 3. Principle of electro-catalytic oxidation

Experiment was carried out as follows: Adjust the $\mathrm{pH}$ of the mixed wastewater to $4.5 \sim 5$, and set the current density of the electrode plate to $5 \mathrm{~mA} / \mathrm{cm} 2,10 \mathrm{~mA} / \mathrm{cm} 2$ and $15 \mathrm{~mA} / \mathrm{cm} 2$, respectively (five parallel experiments were carried out under each current density condition), conduct the batch test under the condition that the reaction time (HRT) was $1 \mathrm{~h}$. The average value of five groups under each current density condition is taken as the COD concentration.

\subsection{Combined Process Pilot-scale Experiment}

According to the results of three kinds of laboratoryscaled experiments, the SBR and electro-catalytic reaction were combined to treat the caprolactam waste- water in pilot scale. The combined process pilot test site is shown in Figure 4. The processing capacity of the combined process was designed as $2 \mathrm{t} / \mathrm{d}$ wastewater, the pilot devices of electro-catalysis and SBR were the main parts, installed and debugged at the test site. In addition to major installation, some assistant equipment was needed to be installed simultaneously, such as the ammoximation wastewater inlet metering pump $(125 \mathrm{~L} / \mathrm{h}, 0.3 \mathrm{MPa}$, suction $3 \mathrm{~m}$, and motor capacity $0.25 \mathrm{~kW})$, inlet metering pump for waste concentrating water/regene- ration water/ammonium sulfate condensate water $(40 \mathrm{~L} / \mathrm{h}, 0.3$ Mpa, suction $3 \mathrm{~m}$, and motor capacity $0.18 \mathrm{~kW}$ ), regulating tank, dosing device, electric control cabinet and so on.

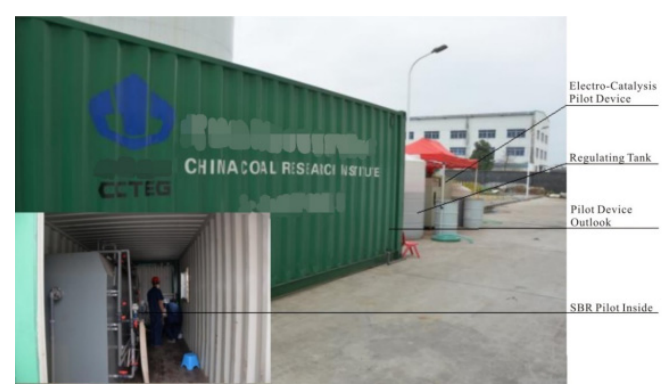

Figure 4. Figure of combined process pilot test site

The pilot-scale experiment was performed as follows: After all the equipment were installed and debugged, collect the activated sludge from the sewage station of the chemical plant, the inoculum size of sludge was 5000 $\mathrm{mg} / \mathrm{L}$, and domesticate the activated sludge for about 20 days. Let the system stabilize, start the pilot test. The flowchart of combined process is shown in Figure 5. The devices were continuously operated without interruption. During the operation, the water sample was taken every 8 hours to test its COD, and the COD concentration in water of each day was the average value of the COD from three water samples.

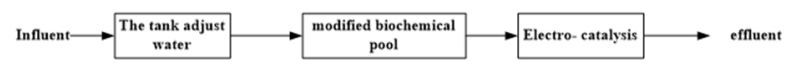

Figure 5. Flowchart of combined process pilot test

\section{Results and discussion}

Figure 6 shows the influence of oxidant dosage on the COD degradation of wastewater, under the conditions that the $\mathrm{pH}$ of wastewater was adjusted to 3.5-4.0 and 3\%o (mass fraction) FeSO4 was as the catalyst. When the oxidant $\mathrm{H} 2 \mathrm{O} 2$ dosage is lower than $2 \%$ of the total volume of wastewater, although the COD has a downward trend, the decrease is not significant due to oxidant dosage is too low and oxidation intensity is not enough. As the dosage of oxidant increased, the reaction intensity increased. Therefore when the $\mathrm{H} 2 \mathrm{O} 2$ dosage increased to $3 \%$, the decrease of COD concentration was obvious, and the COD concentration downed to about $700 \mathrm{mg} / \mathrm{L}$.

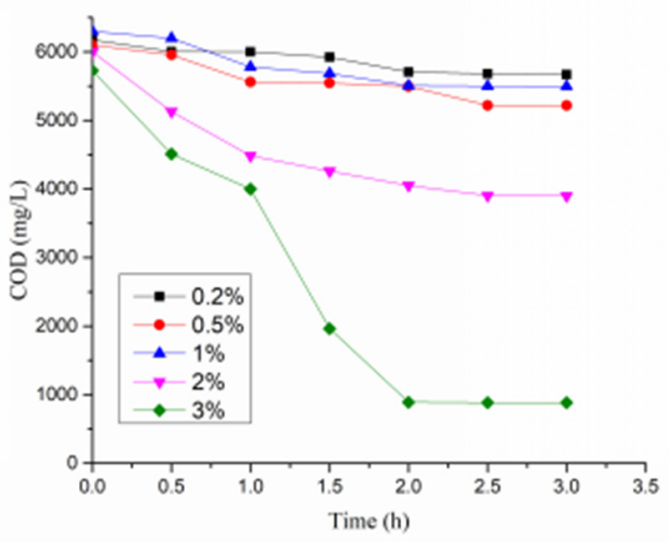

Figure 6. Effect of oxidant $\mathrm{H}_{2} \mathrm{O}_{2}$ dosage on COD degradation 


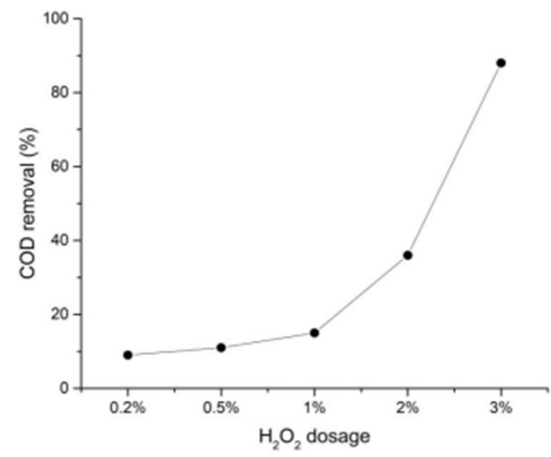

Figure 7. Relationship between the dosage of oxidant $\mathrm{H}_{2} \mathrm{O}_{2}$ within a certain range and COD removal

Figure 7 shows the relationship between the dosage of oxidant $\mathrm{H} 2 \mathrm{O} 2$ within a certain range and $\mathrm{COD}$ removal. It indicated that the COD removal increased with the increase of oxidant $\mathrm{H} 2 \mathrm{O} 2$ dosage when oxidant dosage was from $0.2 \%$ to $3 \%$, and the maximum removal reached to about $90 \%$.

Figure 8 shows the effect of SBR treating wastewater, under the condition that the biochemical influent water quality remained steady, the activated sludge could maintain a high removal rate of organic matter after acclimation, and the CODcr of organic wastewater was degraded from $4254 \mathrm{mg} / \mathrm{L}$ to below $2450 \mathrm{mg} / \mathrm{L}$. Depending on the $33 \mathrm{~d}$ monitoring data, the removing efficiency of CODcr was not only kept steady (left picture in Figure 8) but also increasing slowly (right picture in Figure 8), thereby further stating that the capacity of removing organism of activated sludge was enhanced as time continues. And had been calculated, the removing rate could reach $56.09 \%$.

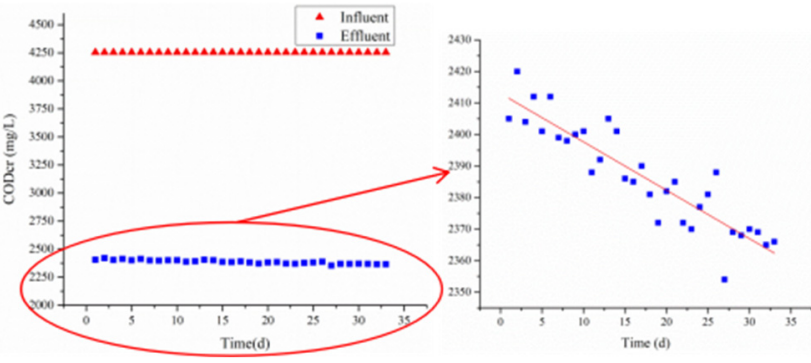

Figure 8. Change of effluent CODcr concentration with time when the CODcr concentration of influent was same

Table 2. Variations in CODcr at different ampere density

\begin{tabular}{|c|c|c|c|c|}
\hline $\begin{array}{c}\text { Ampere } \\
\text { density }\end{array}$ & $\begin{array}{c}\text { Inflow } \\
\text { water } \\
\text { CODcr } \\
\text { (mg/L) }\end{array}$ & $\begin{array}{c}\text { Discharged } \\
\text { water } \\
\text { CODcr } \\
\text { (mg/L) }\end{array}$ & $\begin{array}{c}\text { Removal } \\
\text { efficiency }\end{array}$ & $\begin{array}{c}\text { Power } \\
\text { consumptio } \\
\text { n water per } \\
\text { ton }\end{array}$ \\
\hline $\begin{array}{c}5 \\
\mathrm{~mA} / \mathrm{cm}^{2}\end{array}$ & 4355 & 3484 & $20.0 \%$ & 7.5 \\
\hline $\begin{array}{c}10 \\
\mathrm{~mA} / \mathrm{cm}^{2}\end{array}$ & 4254 & 2405 & $43.5 \%$ & 15 \\
\hline $\begin{array}{c}15 \\
\mathrm{~mA} / \mathrm{cm}^{2}\end{array}$ & 4503 & 1781 & $60.5 \%$ & 22.5 \\
\hline
\end{tabular}

Table 2 and Figure 9 demonstrate COD removal rate at different ampere densities. According to them, it can be obviously seen that as the ampere density increases, the COD removal rate increases. And when ampere density increased from $5 \mathrm{~mA} / \mathrm{cm} 2$ to $15 \mathrm{~mA} / \mathrm{cm} 2$, the COD removal rate enhanced from $20.0 \%$ to $60.5 \%$. However, the rising ampere density would cause the increase of power consumption of treating wastewater per ton. According to Table 2, when ampere density increased from $5 \mathrm{~mA} / \mathrm{cm} 2$ to $10 \mathrm{~mA} / \mathrm{cm} 2$, the power consumption of treating wastewater per ton doubled. Therefore, the cost of treating caprolactam wastewater by this method alone is high.

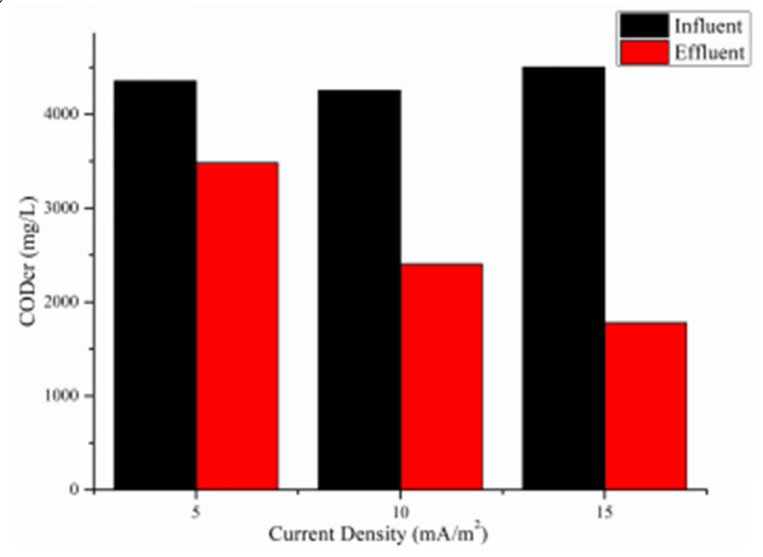

Figure 9. CODcr concentration in influent and effluent at different ampere densities

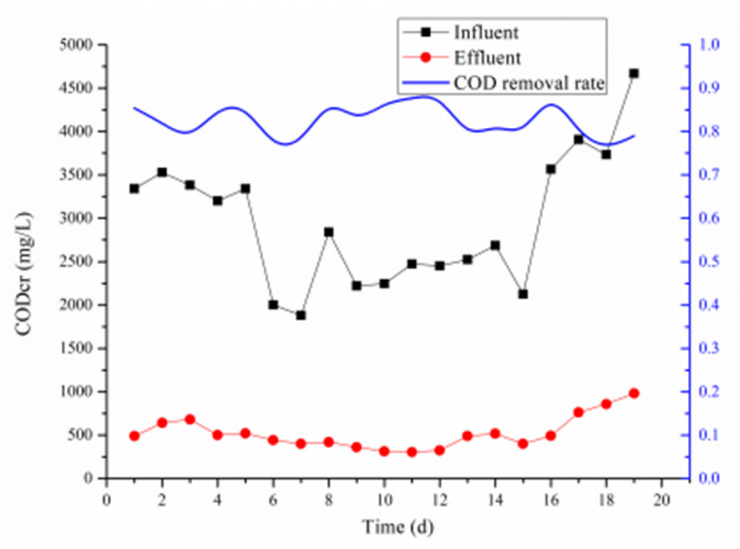

Figure 10. Change of CODcr under the condition of SBR pilot scale test

Figure 10 shows the change of CODcr in caprolactam wastewater under the condition of SBR pilot scale test. It can be seen from Figure 10 that the concentration of CODcr in influent water was unstable and fluctuated (black line), the highest concentration of CODcr in influent was $4669.3 \mathrm{mg} / \mathrm{L}$, and the lowest was 1881.1 $\mathrm{mg} / \mathrm{L}$, which was consistent with the analysis of the source of contaminants in the water samples, and the fluctuation of water quality was determined by the production conditions. The concentration of CODcr in effluent (red line) was stable, and the range was from $303 \mathrm{mg} / \mathrm{L}$ to $980 \mathrm{mg} / \mathrm{L}$, which satisfied the drainage requi- rements of the wastewater treatment plant in the industrial park for the incorporated enterprises, that is, the CODcr is generally not higher than $1000 \mathrm{mg} / \mathrm{L}$. The CODcr removal 
rate (blue line) stabilized around $80 \%$, changed from $77 \%$ to $87.7 \%$.

Figure 11 shows the effect of combined process pilot scale test by SBR and electro-catalytic on the treating of caprolactam wastewater When the concentration of CODcr in influent water changed from $2000 \mathrm{mg} / \mathrm{L}$ to 4000 $\mathrm{mg} / \mathrm{L}$, the discharged water quality was stable and electrocatalytic process could remove refractory organisms with high efficiency. It was found that Electro- catalytic could not only remove organisms but also obviously removed chroma because the base material of three-dimensional catalytic materials (activated carbon) indicated strong adsorption capacity on organisms and pigments. Thereby during the pilot-scale experiment, the equipment of electro-catalytic was designed after SBR, and the data demonstrated that electro-catalytic could considerably degrade organism of biochemically dischar- ged water. The final removal rate of CODcr could be $52 \%-70 \%$, and the concentration of CODcr could be decreased to 104 $\mathrm{mg} / \mathrm{L}$.

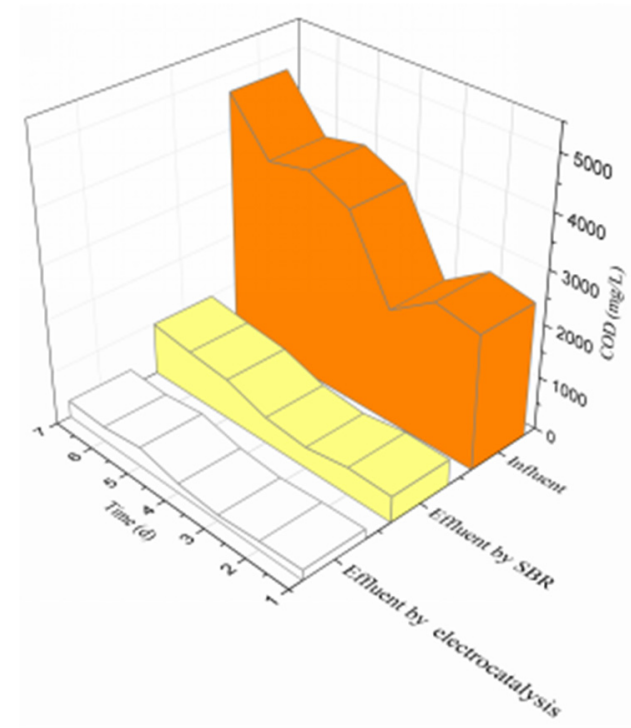

Figure 11. The effect of combined process pilot scale test by SBR and electro-catalytic

According to the experimental results, irrespective of the amount of oxidant dosage, the reaction tends to be stable after $2 \mathrm{~h}$. Wang et al.'s study (Wang et al., 2014) also showed that the dosage of oxidant would affect the treatment of caprolactam wastewater. They found that using $\mathrm{H} 2 \mathrm{O} 2$ as oxidants, the $\mathrm{COD}$ removal rate could reach $70 \%$ under suitable reaction conditions. And the rising ampere density would cause the increase of power consumption of treating wastewater per ton, the cost of treating caprolactam wastewater by SBR treating alone is high. A comparison between sample experiment results with those of pilot-scale experiment indicated that pilotscale's removal efficiency was obviously higher than sample experiment, and the process was more stable. Two reasons may contribute to the result. On one side, the expansion of water treatment scale provided more stable water ecosystem, which was conducive for microorganisms. On the other hand, activated sludge of sample experiment was collected from sanitary sewage, the pilot-scale experiment was inoculated with activated sludge from the owned wastewater treatment plant of the chemical plant, and the latter was significantly stronger after long-term domestication, which had been cultivated and improved removal efficiency. So the equipment of electro-catalytic was designed after SBR. And the data demonstrated that electro-catalytic could considerably degrade organism of biochemically discharged water.

The power consumption of treating wastewater per ton was approximately $5.72 \mathrm{kWh} / \mathrm{m} 3$, when the electric charge was $0.7 \mathrm{CNY} / \mathrm{kWh}$, the system power consumption was $4 \mathrm{CNY} / \mathrm{m} 3$. The water consumption (equipment cleaning, dispense medicines, and experiment) of the system was $0.04 \mathrm{CNY} / \mathrm{m} 3$. The medicament consumption was $0.66 \mathrm{CNY} / \mathrm{m} 3$, including flocculant (bit), coagulant (bit), and neutralizing agent (vast). The transportation expenses of surplus sludge (moisture content $<80 \%$ ) were $200 \mathrm{CNY} / \mathrm{t}$ (haul distance $\leq 20 \mathrm{~km}$ ), and the corrected fee was $0.18 \mathrm{CNY} / \mathrm{m} 3$.Corrected staff salary was 0.27 $\mathrm{CNY} / \mathrm{m} 3$. The consumption of operation and maintenance was calculated as $10 \%$ of total consumption. The finally total consumption of wastewater treatment was approximately $5.67 \mathrm{CNY} / \mathrm{m} 3$.

\section{Conclusion}

(1) The removal rate of Fenton depended on the dosage of $\mathrm{H} 2 \mathrm{O} 2$. When the dosage was below $1 \%$, the rate was not obvious $(<20 \%)$. However, when the dosage increased to $3 \%$, the rate reached $90 \%$. However, the consumption of $\mathrm{H} 2 \mathrm{O} 2$ was huge, corrected economic depletion of medicament was $6 \mathrm{CNY} / \mathrm{m} 3$, and the total fee may exceed $10 \mathrm{CNY} / \mathrm{m} 3$. This process resulted in a considerable financial burden for manufacturing enterprises.

(2) The research confirmed that caprolactam wastewater can be treated by biochemistry approach. The SBR test indicated that the removal rate was $56.09 \%$. At the pilot-scale experiment, the SBR reactor indicated improved removal rate (above 50\%). However, single SBR indicated difficulties in meeting the requirement of current environmental protection. Thus, the approach must be combined with oxidation technology.

(3) The electro-catalytic process can further address the discharged water of SBR. The removal rate could be $52-70 \%$, and the final CODcr can be decreased to $104 \mathrm{mg} / \mathrm{L}$.

(4) Applying the combined process can not only remove organism of caprolactam wastewater with high efficiency, but it also maintains good economic feasibility. This study demonstrated that the SBR electro-catalytic combined process could treat caprolactam wastewater with $90 \%$ removal rate. The economical consumption could be controlled under $6 \mathrm{CNY} / \mathrm{m} 3$.

\section{Acknowledgments}

Financial support for this research was provided by the National Key R\&D Program of China (Grant No.2017YFB0602304). This work also supported by 
Shenzhen Science and Technology Program (Grant No. JCYJ20150529114024234), the National Key Research and Development Program of China (No.2017YFB0602 304).

\section{References}

1. X. Chen, Z. Zhang, Z Li, Technol. Water Treat., 05(2013).

2. F. Li, J.H. Chen, Q. Chen, Q. Yuan, J. Donghua Univ., 32, 108(2006).

3. N. Gedam, N. R. Neti, S. M. Kashyap. CLEAN - Soil Air Water, 42, 932(2014).

4. J. Dosta, S. Lo' pez-Palau, J. Mata-A' lvarez. Water Sci. Technol, 58, 119(2008).

5. L. Dong, Z. Zhang, J. Gao, W. Guan. Environ. Prot. Chem. Ind., 25, 121(2005).

6. P. Chen, S. Zhang, N. Lin, W. Chen. J. Yangtze Univ., 16(2016).

7. S. Du, J.K. Wang, W.G. Dong, M.L. Gao, F. Duan, C.R. Fu. Environ. Prot. Xinjiang., 37,27(2015).

8. A.Ž. Gotvajn,, J. Zagorc-Končan,, M. Cotman. Desalination., 275, 269(2011).

9. A. Dhaouadi, L. Monser, N. Adhoum. Electrochim. Acta., 54, 4473(2009). 